\title{
WELL-POSEDNESS OF A DISSIPATIVE SYSTEM MODELING ELECTROHYDRODYNAMICS IN LEBESGUE SPACES
}

\author{
Jihong Zhao, Chao Deng And Shangbin Cui
}

\begin{abstract}
In this paper, we study a dissipative system of partial differential equations modeling the flow of electrohydrodynamics. This system consists of the Navier-Stokes equations with a source term coupled with the Nernst-Planck-Poisson equations for electronic charges. We establish local well-posedness of the initial value problem of this system in the critical and subcritical vector Lebesgue spaces. Moreover, we also prove that if the initial data is sufficiently small in critical Lebesgue spaces, then the solution is a global.
\end{abstract}

Mathematics subject classification (2010): 35K15, 35K55, 35Q35.

Keywords and phrases: dissipative system, Navier-Stokes equations, Nernst-Planck-Poisson equations, well-posedness.

\section{REFERENCES}

[1] N. Ben Abdallah, F. Méhats And N. Vauchelet, A note on the long time behavior for the drift-diffusion-Poisson system, C. R. Math. Acad. Sci. Paris, 339, 10 (2004), 683-688.

[2] P. Biler And J. Dolbeault, Long time behavior of solutions to Nernst-Planck and Debye-Hückel drift-diffusion systems, Ann. Henri Poincaré, 1 (2000), 461-472.

[3] P. Biler, W. Hebisch And T. NADZIEJA, The Debye system: existence and large time behavior of solutions, Nonlinear Anal., 23 (1994), 1189-1209.

[4] J. Bourgain AND N. PAVlović, Ill-posedness of the Navier-Stokes equations in a critical space in 3D, J. Funct. Anal., 255 (2008), 2233-2247.

[5] P. B. Balbuena And Y. WANG, Lithium-ion Batteries, Solid-electrolyte Interphase, Imperial College Press, 2004.

[6] M. CAnnone, A generalization of a theorem by Kato on Navier-Stokes equations, Rev. Mat. Iberoam., 13 (1997), 515-541.

[7] P. DeBYe AND E. HÜCKEL, Zur Theorie der Elektrolyte, II: Das Grenzgesetz für die elektrische Leiffähigkeit, Phys. Z., 24 (1923) 305-325.

[8] E. T. ENiKov AND B. J. Nelson, Electrotransport and deformation model of ion exchange membrane based actuators, Smart Structures and Materials, Vol. 3978 (2000), 129-139.

[9] E. T. ENIKOV AND G. S. SEO, Analysis of water and proton fluxes in ion-exchange polymer-metal composite (IPMC) actuators subjected to large external potentials, Sensors and Actuators, 122 (2005), 264-272.

[10] H. Fujita And T. Kato, On the Navier-Stokes initial value problem I, Arch. Rational Mech. Anal., 16 (1964), 269-315.

[11] Y. GigA, Solutions for semilinear parabolic equations in $L^{p}$ and regularity of weak solutions of the Navier-Stokes system, J. Differential Equations, 62 (1986), 186-212.

[12] H. GAJEWSKI AND K. GRÖGER, On the basic equations for carrier transport in semiconductors, J. Math. Anal. Appl., 113 (1986), 12-35.

[13] J.W. Jerome, Analytical approaches to charge transport in a moving medium, Tran. Theo. Stat. Phys., 31 (2002), 333-366.

[14] J. W. JEROME AND R. SACCO, Global weak solutions for an incompressible charged fluid with multiscale couplings: Initial-boundary-value problem, Nonlinear Anal., 71 (2009), 2487-2497.

[15] G. KARCH, Scaling in nonlinear parabolic equations, J. Math. Anal. Appl., 234 (1999), 534-558. 
[16] G. KARCH, Scaling in nonlinear parabolic equations: applications to Debye system, AIP Conf. Proc., 553(1) (2001), 243-248.

[17] T. KATO, Strong $L^{p}$ solutions of the Navier-Stokes equations in $\mathbb{R}^{m}$ with applications to weak solutions, Math. Z., 187 (1984), 471-480.

[18] T. Kato AND G. Ponce, Well-posedness of the Euler and Navier-Stokes equations in the Lebesgue spaces $L_{p}^{s}\left(\mathbb{R}^{2}\right)$, Rev. Mat. Iberoam., 2 (1986), 73-88.

[19] T. Kato And G. Ponce, Commutator estimates and the Euler and Navier-Stokes equations, Comm. Pure Appl. Math., 41 (1988), 891-907.

[20] H. Koch AND D. TATARU, Well-posedness for the Navier-Stokes equations, Adv. Math., 157 (2001), $22-35$.

[21] J. LERAY, Sur le mouvement d'un liquide visqueux emplissant l'espace, Acta Math., 63 (1934), $193-$ 248.

[22] M. Longaretti, B. Chini, J.W. Jerome And R. Sacco, Electrochemical modeling and characterization of voltage operated channels in nano-bio-electronics, Sensor Letters, 6 (2008), 49-56.

[23] M. Longaretti, B. Chini, J.W. Jerome And R. S ACCo, Computational modeling and simulation of complex systems in bio-electronics, J. Computational Electronics, 7 (2008), 10-13.

[24] M. Longaretti, G. Marino, B. Chini, J.W. Jerome and R. S Acco, Computational models in nano-bio-electronics: simulation of ionic transport in voltage operated channels, J. Nanoscience and Nanotechnology, 8 (2008), 3686-3694.

[25] F. Planchon, Global strong solutions in Sobolev or Lebesgue spaces to the incompressible NavierStokes equations in $\mathbb{R}^{3}$, Ann. Inst. Henri Poincare, 13 (1996), 319-336.

[26] I. RUbinsteIn, Electro-Diffusion of Ions, SIAM Studies in Applied Mathematics, SIAM, Philadelphia, 1990.

[27] R. J. RYHAM, Existence, uniqueness, regularity and long-term behavior for dissipative systems modeling electrohydrodynamics, arXiv:0910.4973v1.

[28] R. J. RYham, C. LiU AND L. ZikATANOV, Mathematical models for the deformation of electrolyte droplets, Discrete Contin. Dyn. Syst. Ser. B, 8, 3 (2007), 649-661.

[29] M. SCHMUCK, Analysis of the Navier-Stokes-Nernst-Planck-Poisson system, Math. Models and Methods Appl. Sci., 19, 6 (2009), 993-1015.

[30] M. SHAHINPOOR AND K. J. KIM, Ionic polymer-metal composites: III. Modeling and simulation as biomimetic sensors, actuators, transducers, and artificial muscles, Smart Mater. Struct., 13 (2004), $1362-1388$.

[31] R. Temam, Navier-Stokes equations, AMS Chelsea Publishing, Providence, RI, (2001). Theory and numerical analysis, Reprint of the 1984 edition. 